\title{
ULTRAVIOLET TO NEAR INFRARED OBSERVATIONS OF BF Cyg
}

A. Cassatella, R. Gonzalez-Riestra

IUE Observatory, ESA, P.O. Box 54065, Madrid

T. Fernandez-Castro

Planetario de Madrid, Madrid, Spain

J. Fuensalida

Instituto de Astrofisica de Canarias, La Laguna

A. Gimenez

Instituto de Astrofisica de Andalucia, Granada

In this paper we provide preliminary results of multifrequency observations of BF Cyg carried out in July 1986. The ultraviolet spectra were obtained on July 26, 1986 using the IUE satellite. The optical observations were made at the Observatorio del Roque de los Muchachos (La Palma, Canary Islands) in July 1986 during the night 13/14 using the Isaac Newton $2.5 \mathrm{~m}$ telescope with the Intermediate Dispersion Spectrograph (IDS, $500 \mathrm{~mm}$ camera) and the Image Photon Counting system (IPCS). The infrared observations were made during the night $13 / 14$ of July, 1986 , at the Observatorio del Teide (Tenerife, Canary Islands) using the Carlos Sanchez $1.5 \mathrm{~m}$ telescope and an infrared single-channel photometer with an Insb detector.

In figure 1 , the combined ultraviolet and optical energy distribution of BF Cyg on July 26, 1986, is plotted from 1150 to $6750 \mathrm{~A}$. In figure 2, the same information is given in logarithmic scale after correction for reddening assuming a colour excess of $\mathrm{E}(\mathrm{B}-\mathrm{V})=0.35$. A preliminary analysis of the observed spectrum allows one to identificy three well-defined components in BF Cyg (see Fig. 2):

a) A hot ionizing component, with Teff > $60000 \mathrm{~K}$ can be deduced from the strength of the HeII 1640 A line. This source, which dominates the spectrum below about $2000 \mathrm{~A}$, is most probably a hot subdwarf.

b) A ionized nebula surrounding the hot source or the whole system, responsible for the conspicuous Balmer recombination continuum in emission easily visible in the range 2600 to $3600 \mathrm{~A}$.

c) A cool giant with an effective temperature of about $3000 \mathrm{~K}$ which dominates the spectrum in the near infrared. 


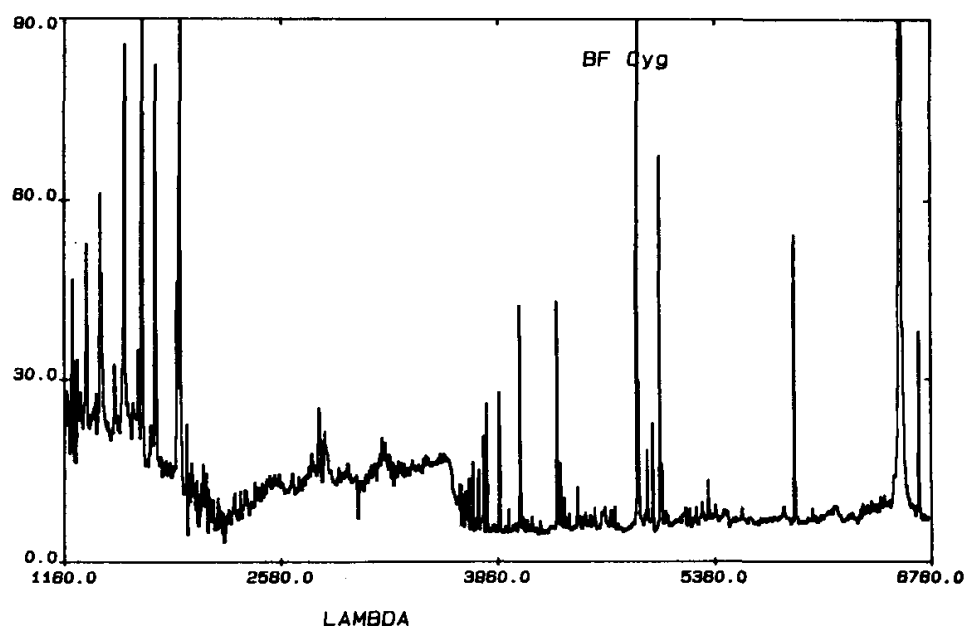

Figure 1. Oberved energy distribution of $B F$ Cyg in the ultraviolet and optical regions.

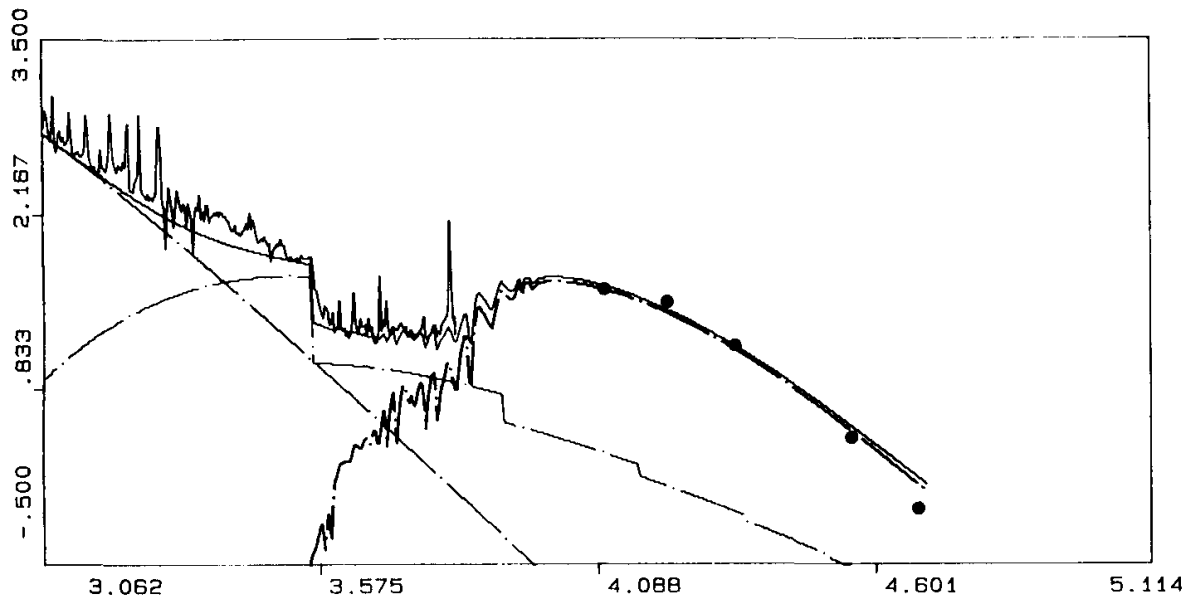

Figure 2. Preliminary model of the energy distribution of $\mathrm{BF}$ Cyg. Fluxes were corrected for reddening using $E(B-V)=$ 0.35. The energy distribution is consistent with the presence of three components: a) a hot source represented by a black body with $T=80000 \mathrm{~K}$; $\mathrm{b})$ hydrogen $f-b$ and $f-f$ continuum emission with $\mathrm{T}_{\mathrm{l}} \mathrm{l}=20000 \mathrm{~K} ; \mathrm{cl}$ a cool component, represented with a standard M5III star (shortward of $10000 \mathrm{~A}$ ) and with a $3000 \mathrm{~K}$ black-body (at longer wavelengths). 